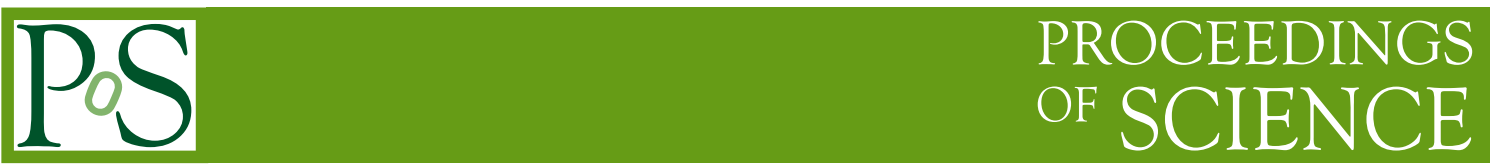

\title{
Physics with the CMS forward CASTOR calorimeter
}

\section{Igor Katkov for the CMS Collaboration}

DESY, Germany and Moscow State University, Russia

E-mail: katkov@mail.desy.de

The CASTOR calorimeter is designed for the very forward region (pseudorapidity range from -6.6 to -5.2$)$ in the CMS experiment at LHC. It will be operated in the low luminosity period of $p p$-collisions up to $2 \times 10^{33} \mathrm{~cm}^{-2} \mathrm{~s}^{-1}$ and in heavy-ion-collisions. The design of the calorimeter is determined by space constraints inside a shield for radiation and for magnetic field and restricted to materials which tolerate a high radiation level. The calorimeter surrounds the beam pipe as a very compact sampling structure of tungsten and quartz plates with a depth of 10 hadronic interaction lengths. The granularity of 16 transversal segments and 14 longitudinal sections allows to reconstruct shower profiles, to separate electrons and photons from hadrons and to search for phenomena with anomalous hadronic energy depositions as expected from exotica. Performance as measured in test beams, first operation experience and first measurements in the very forward region of CMS will be presented.

The XIXth International Workshop on High Energy Physics and Quantum Field Theory, QFTHEP2010 September 08-15, 2010

Golitsyno, Moscow, Russia 


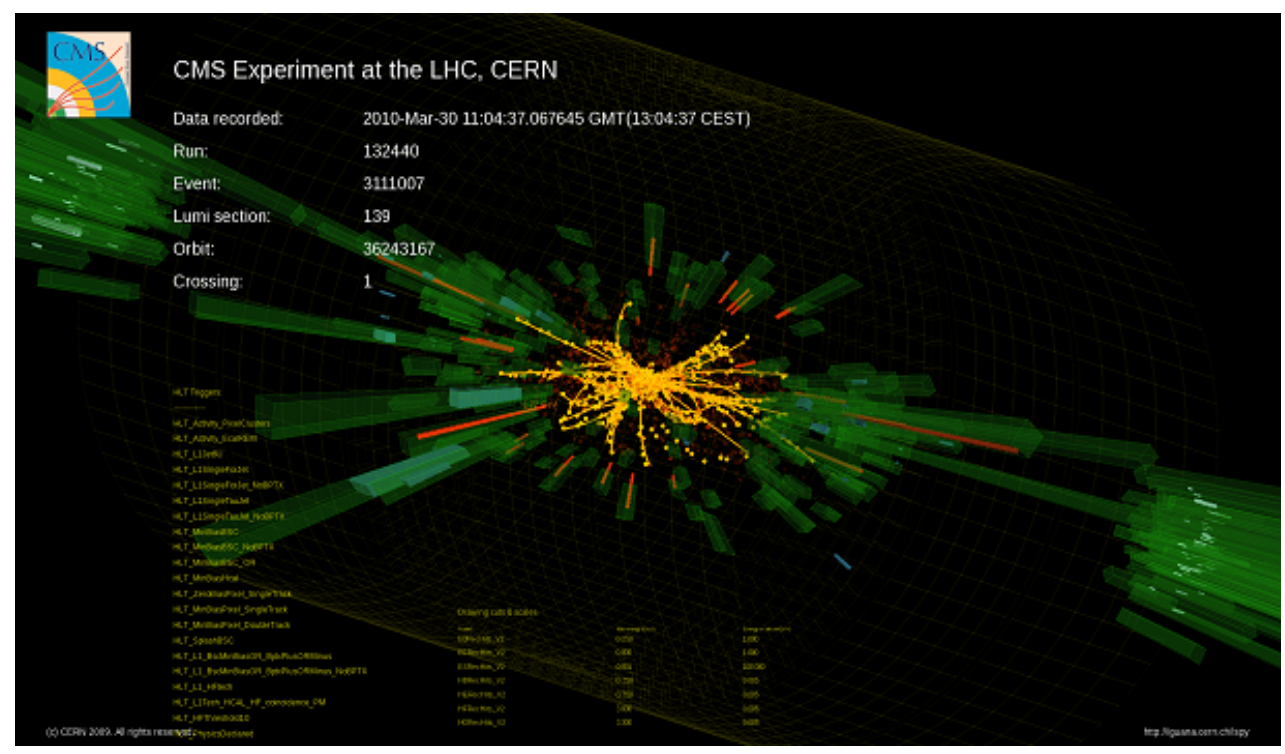

Figure 1: One of the first collision event at $7 \mathrm{TeV}$ centre of mass energy as seen in the CMS event display

\section{CASTOR as a forward calorimeter of the CMS}

The CMS detector [1] is built around its solenoid with a strong magnetic field of 3.8T. Such a magnetic field is both a big advantage and a challenge for detector operation and in particular in the forward region of the detector. It is in this region where the forward CASTOR calorimeter is installed on the platform of the Hadronic Forward (HF) calorimeter.

The CMS had been collecting high quality data at $7 \mathrm{TeV}$ centre of mass energy since March 2010. In Fig. 1 an event display of one of the first collisions is shown. By the time of this report the CMS had collected well above $3 \mathrm{pb}^{-1}$ of integrated luminosity as shown in Fig. 2. In the beginning of September 2010 the LHC was running with 50 bunches, whereas 35 bunches were colliding in ATLAS and CMS. The LHC provided experiments with conditions for a steep and steady increase of their data samples. Bunch train commissioning was under way implying smaller bunch separation in time and hence more strict requirements on time response of detector components.

For trigger purposes, the two-step CMS trigger system is used together with two elements of the CMS detector monitoring system, the Beam Scintillation Counters (BSC) and the Beam Pick-up-Timing for the experiments (BPTX) devices. The BPTX provides precise information on the structure and timing of LHC beams. The coincidence of signals from both BPTX detectors indicates the presence of both beams passing the interaction point. The BSC comprises two sets of scintillation tiles installed in front of the HF (BSC1) and CASTOR (BSC2) calorimeters providing information on hits and coincidence signals. The minimum bias, beam halo, beam gas, beam splash, and high multiplicity triggering as well as the beam condition monitoring in proton-proton and heavy ion collisions are based on the information from the BPTX and BSC devices. The BSC2 in particular was used for triggering in special CASTOR calibration runs.

The CASTOR project involves groups from Belgium (Antwerp), Brazil (UERJ), CERN, DESY, Greece (Athens), Russia (JINR, INR, ITEP, MSU), Turkey (Adana, Cukurova), USA (Northeastern). The project history in CMS has started in 2003. In 2007 CASTOR was officially approved 


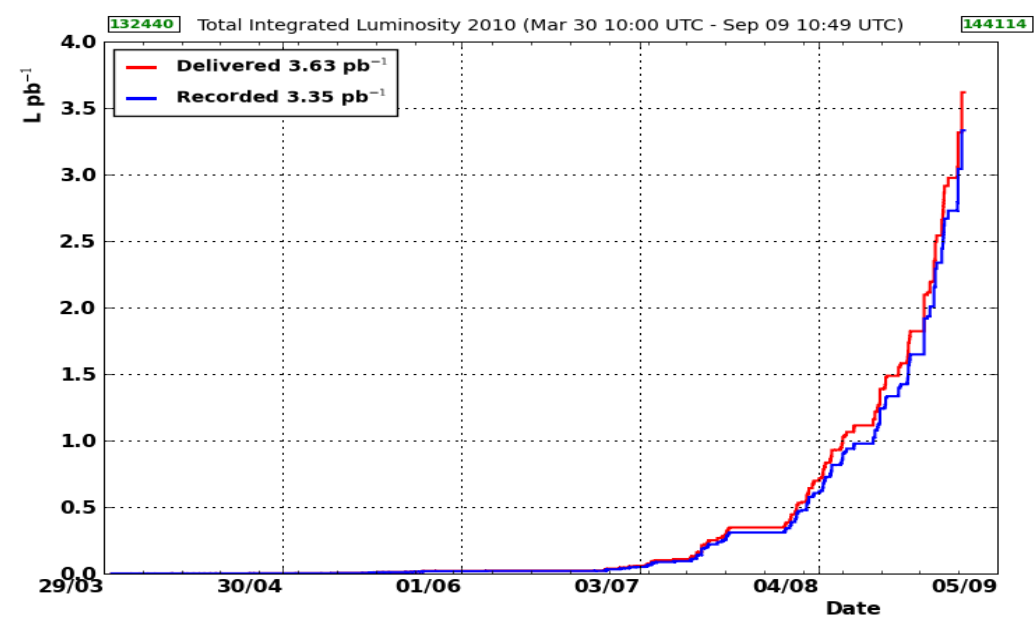

Figure 2: The integrated luminosity delivered to and recorded by CMS up until beginning of September 2010

as a CMS component. Test beams of full-length prototypes were performed at a CERN SPS beam line in 2007, 2008, and 2009. Production of detector components was started in 2008. Also the first installation in the CMS experimental cavern was exercised in 2008.

The detector design is presented in Fig. 3. Conditions in the forward region of the CMS experiment require compact detector dimensions, radiation hardness $(\leq 20 \mathrm{kGy}$ in years 2009 2010), fast response, and operation in presence of magnetic field $(\leq 0.16 \mathrm{~T})$. Tungsten as an absorber and fused silica quartz as an active material satisfy these requirements [2]. CASTOR has 16 azimuthal sectors (semi-octants) which are mechanically organized in two half calorimeters embracing the LHC beam-pipe from two sides. Every sector is longitudinally divided into an electromagnetic section with 2 readout units and a hadronic one with 12 readout units. Such a

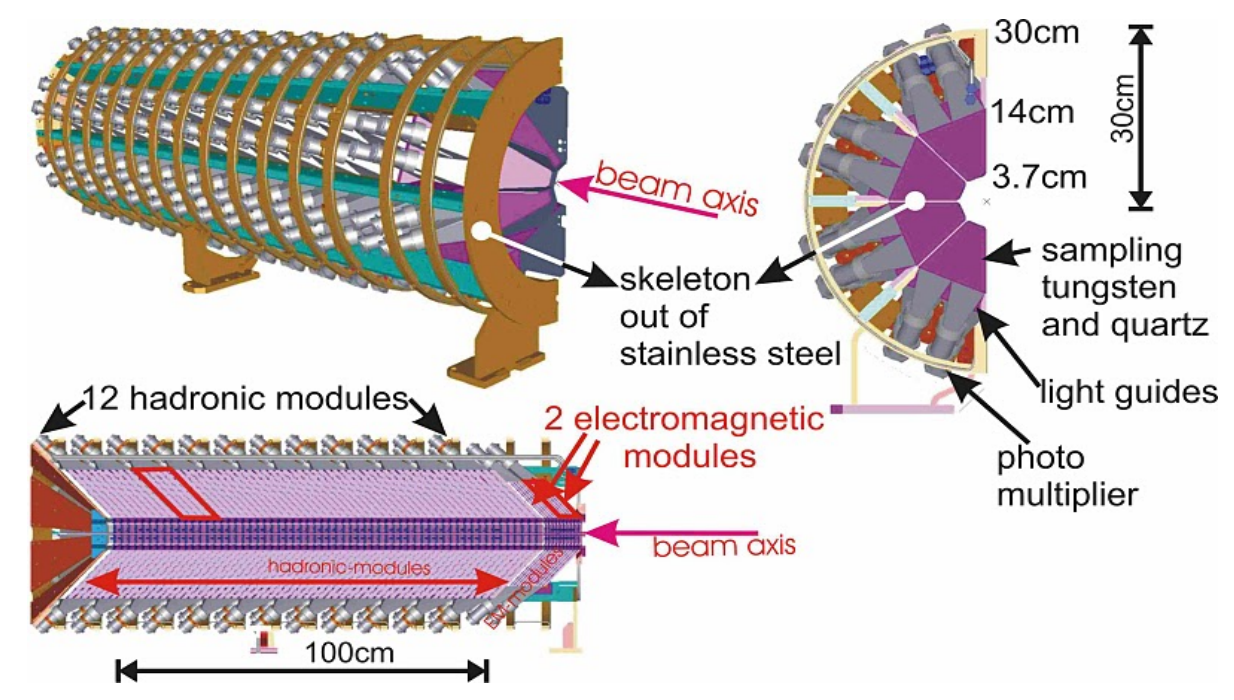

Figure 3: Sketches of the CASTOR calorimeter design. 


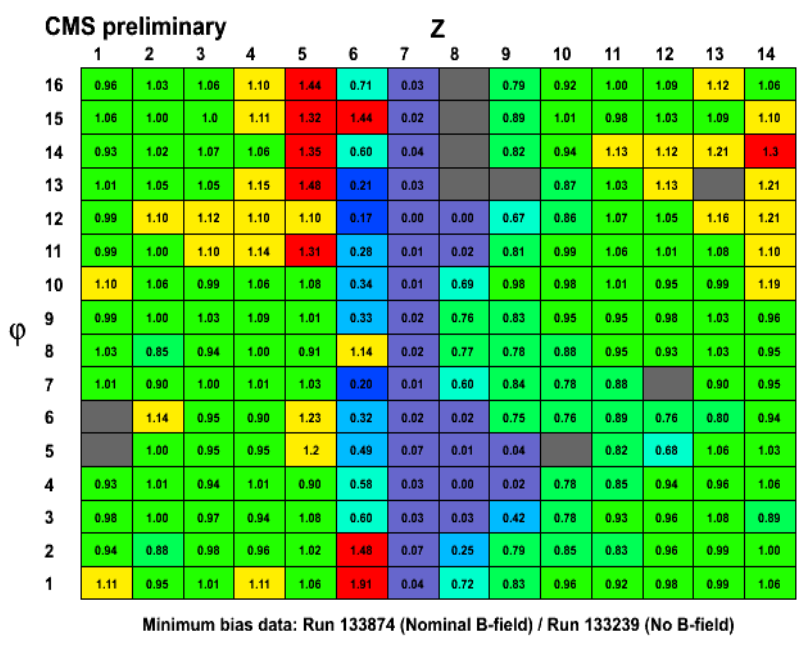

Figure 4: Ratio of average channel response in minimum bias dataset with magnetic field of $\mathrm{B}=3.8 \mathrm{~T}$ and $\mathrm{B}=0 \mathrm{~T}$.

fine granularity allows reconstruction of shower profiles, separation of electrons and photons from hadrons and search for phenomena with anomalous hadronic energy depositions. Pairs of tungsten and quartz plates constitute a sampling unit. Each readout unit consists of 5 sampling units. For the electromagnetic section the tungsten plates are $5 \mathrm{~mm}$-thick, whereas quartz plates are $2 \mathrm{~mm}$ thick. The plates in hadronic readout units have twice the thickness of electromagnetic ones. The stack of plates is inclined by $45^{\circ}$ with respect to the horizontal axis to enhance the collection of the Čerenkov light mixed and guided through tapered air core light guides to photomultipliers converting the light into electrical signals (photomultipliers are inclined by $30^{\circ}$ with respect to the horizontal axis because their orientation is restricted due to presence of the magnetic field). Taking into account the inclination, the amount of material corresponds to 0.77 nuclear interaction lengths (20.12 radiation lengths) in the electromagnetic section and 9.24 nuclear interaction lengths in the hadronic one. Hence the overall depth of the calorimeter is 10 interaction lengths. The total length of the calorimeters is approximately $1.6 \mathrm{~m}$, its diameter is $0.6 \mathrm{~m}$.

The iron shielding in the forward region of CMS has poorly shielded narrow gaps. Fine-mesh PMTs (Hamamatsu R5505), designed to operate in magnetic fields, are used as photodetectors in CASTOR calorimeter. In the region around the gaps the high voltage applied to the PMTs is increased to compensate for the gain loss. The orientation of the PMTs is chosen to account for the presence of the magnetic field. Even so some of calorimeter channels are affected by the stray magnetic field that varies both in magnitude and direction. This is illustrated in Fig. 4 showing the ratio of average channel response in minimum bias dataset with $\mathrm{B}=3.8 \mathrm{~T}$ and $\mathrm{B}=0 \mathrm{~T}$ that quantifies the effect of the magnetic field on calorimeter operation. Modules from $6(3.5 \lambda)$ to 9 $(5.6 \lambda)$ are (partly) affected.

The CASTOR calorimeter uses the design of the CMS hadronic calorimeter readout electronics [3]. The design facilitates implementation of CASTOR-specific trigger conditions and provides wide dynamics range $\left(10^{4}\right)$ needed to measure energy deposits from minimum ionizing particles (calibration) and up to beam energies. Implementation of a muon, energy-threshold, and rapidity- 


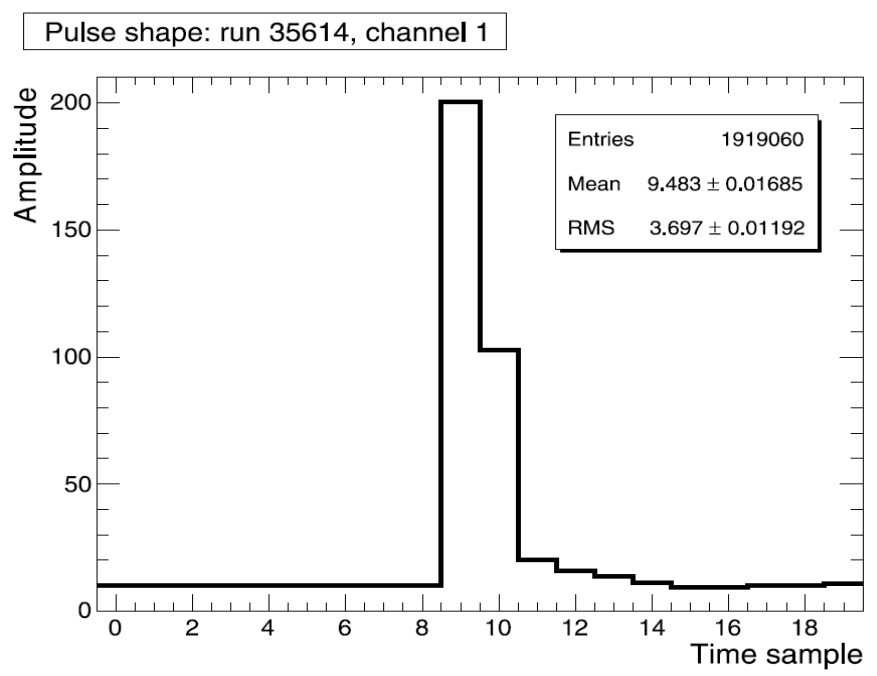

Figure 5: Typical pulse shape sampled in 25-ns time periods as measured in 2007 test beam data.

gap trigger bits based on CASTOR information is foreseen. The PMT signals are sampled in 25-ns time periods as illustrated in Fig. 5 where a typical channel pulse is shown. For running with high intensity LHC beams and triggering the relative phase of calorimeter signals with respect to the LHC clock should be adjusted to get signals entirely within one 25-ns time period.

The design of the calorimeter has been improved in several test beam programs in $\mathrm{H} 2$ beam line of the Super Proton Synchrotron at CERN [4]. Tests were performed with low- and highenergy muon, electron, and pion beams in the range from several GeV to $350 \mathrm{GeV}$. In June 2009 the calorimeter has been installed in the CMS experimental cavern on one side of the CMS detector. It is fully equipped, functional and integrated into CMS operations.

\section{CASTOR performance}

The main parameters of the calorimeter have been studied using prototypes consisting of two full length azimuthal sectors irradiated with test beam particles. A single channel response to $150 \mathrm{GeV}$ muon beam is shown in Fig. 6 overlaid with the pedestal peak. A good signal to noise ratio for muons is essential for relative channel response equalisation, so called intercalibration of the calorimeter. The energy resolution is determined from electron and pion energy scans at the centre of a prototype sector upon selection of a narrow beam spot. The same data were used to check the response linearity. As shown in Fig. 7, the energy resolution, $\sigma / E_{\text {beam }}$, as a function of beam energy can be fitted by the quadratic sum of a stochastic and a constant terms. The energy resolution is around $6 \%$ and $25 \%$ for $100 \mathrm{GeV}$ electrons and pions respectively. The values are reasonable for the harsh conditions in the forward region of the CMS.

To quantify the response uniformity and derive the shower profile properties, prototypes were beam scanned horizontally and vertically with electron and pion beams. The differential profile and the radial containment of the shower were extracted from the data. Full width at half maximum of the differential $x$-profile is $6 \mathrm{~mm}$ as measured for $80 \mathrm{GeV}$ pion beam indicating compact shower dimensions as expected for a Čerenkov calorimeter which is sensitive only to the shower core. 


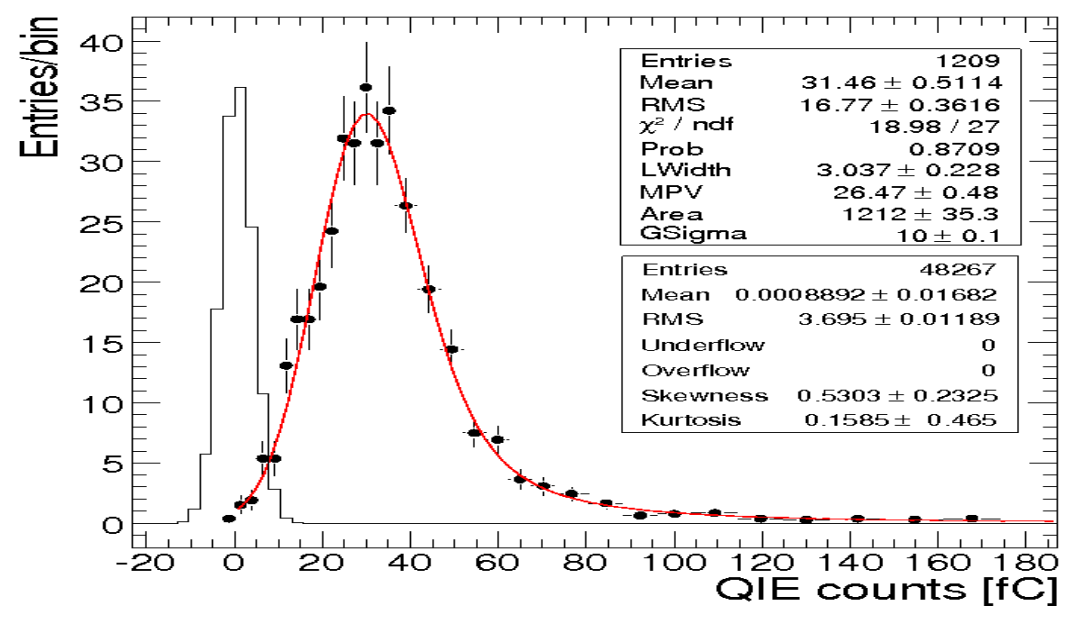

Figure 6: A single channel response to $150 \mathrm{GeV}$ muon beam as measured in 2007 test beam data.
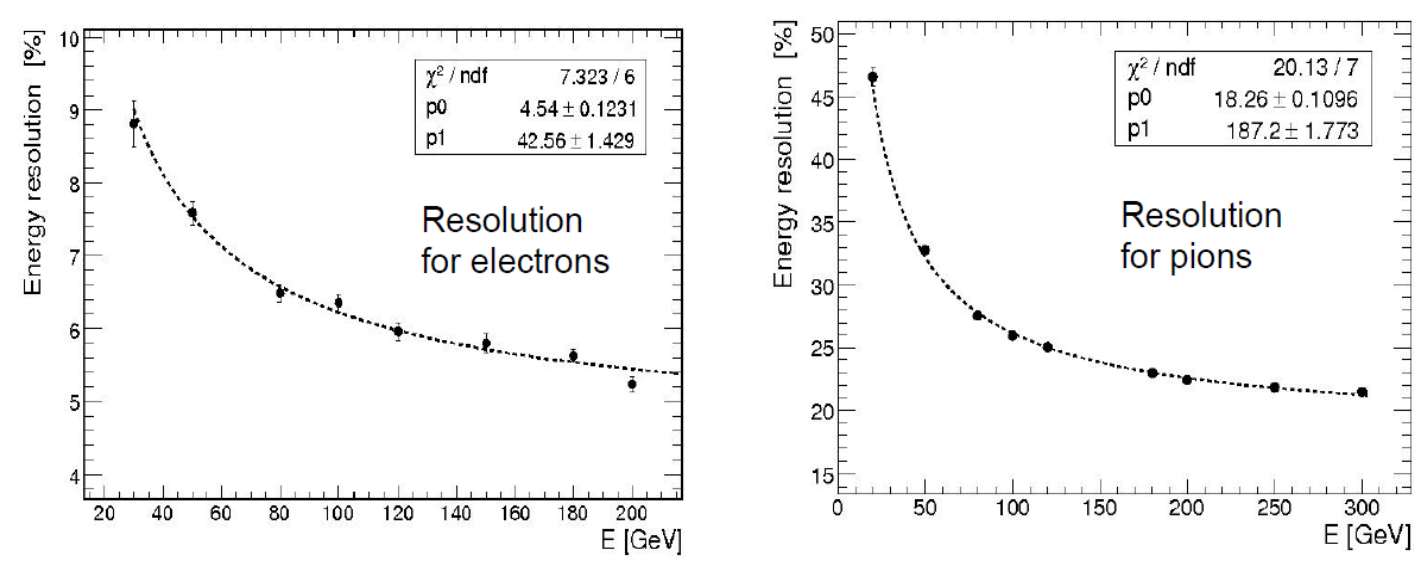

Figure 7: Energy resolution for electrons (left) and pions (right) as measured in 2007 test beam data.

Calibration of a longitudinally segmented calorimeter is known to be a non-trivial task [5]. In situ calibration procedures have to be developed to account for the effect of the magnetic field. Several classes of events are considered to perform the equalisation of channel responses (intercalibration): zero bias and minimum events with calorimeter signal patterns consistent with minimum ionizing particle energy deposits, events collected in special runs during injection and ramp aiming to pick up beam halo muons using the BSC2-based trigger (and later also using own dedicated CASTOR trigger), beam splash events. These different event classes provide opportunity to crosscheck results and possibly combine them to overcome inefficiencies.

Following the analysis strategy adopted by the HF calorimeter, the leptonic decays of $\mathrm{Z}$ bosons can be used to set the absolute electromagnetic energy scale. Significant amount of collected integrated luminosity is needed (roughly estimated to be at least above $10 \mathrm{pb}^{-1}$ ) to perform this task with reasonable precision. The jet energy scale can be set using di-jet, jet-plus-gamma, and Z-plusjet event configurations. Fig. 8 illustrates a good potential of calibration using di-jet events given 

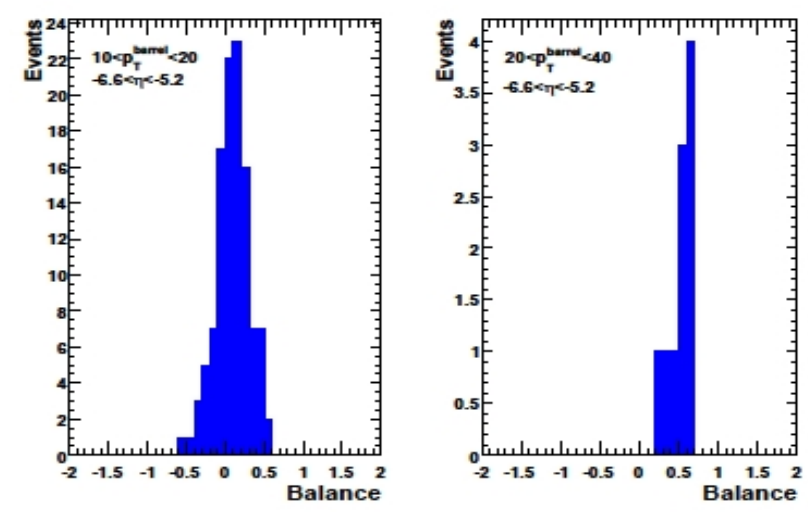

Figure 8: Di-jet balance, $\left(p_{t}[\right.$ probe $]-p_{t}[$ barrel $\left.]\right) / p_{t}[$ barrel $]$, for back-to-back two-jet configuration with a jet in the barrel region and a probe jet in CASTOR in bins of the transverse momentum, $p_{t}>10 \mathrm{GeV}$, of the barrel jet in Monte Carlo simulated events.
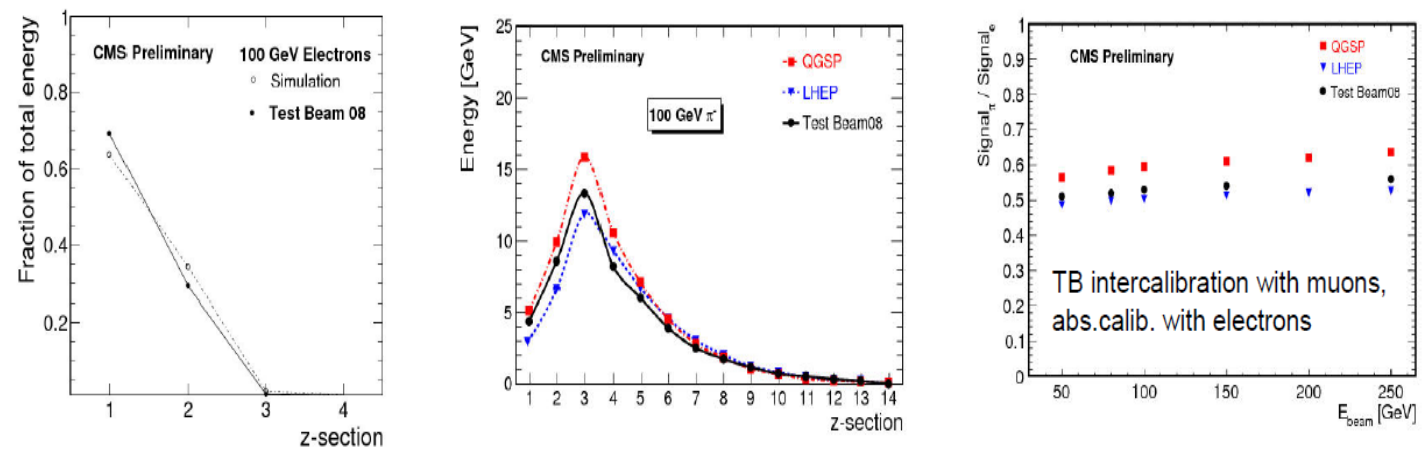

Figure 9: The longitudinal shower profile for electrons (left) and pions (centre) with an energy of $100 \mathrm{GeV}$ as obtained in a beam test set-up is compared to a MC simulation of the detector. The ratio of the mean total collected charge for pions with respect to electrons of the same energy as a function of energy (right) in a beam test set-up and in MC simulations.

the fact that the transverse momentum is still high enough to be triggered in the barrel region and events are numerous. The needs of basic analyses with CASTOR, namely energy flow, underlying event and low- $x$ QCD studies, indicate that calibration precision at $10-20 \%$ level is required.

The CMS global software network includes all essential CASTOR reconstruction and simulation software. CASTOR high level objects, such as jets, reconstructed upon unpacking initial raw data and combination of hits into clusters, are available in the data at Tier 2 centres. Fine tuning of calorimeter full simulation and shower library, which is needed to include the detector simulated data into standard CMS Monte Carlo samples, is well under way. Fig. 9 shows results of validation of full simulation using CASTOR test beam data for longitudinal shower profiles for $100 \mathrm{GeV}$ electrons and pions, and for calorimeter non-compensation.

The CASTOR trigger is crucial to improve efficiency of in situ calibration procedures and physics event selection. Upon implementation of CASTOR trigger conditions as simple CMS technical trigger bits in the longer term they will be added to existing trigger algorithms to avoid prescale factors. 

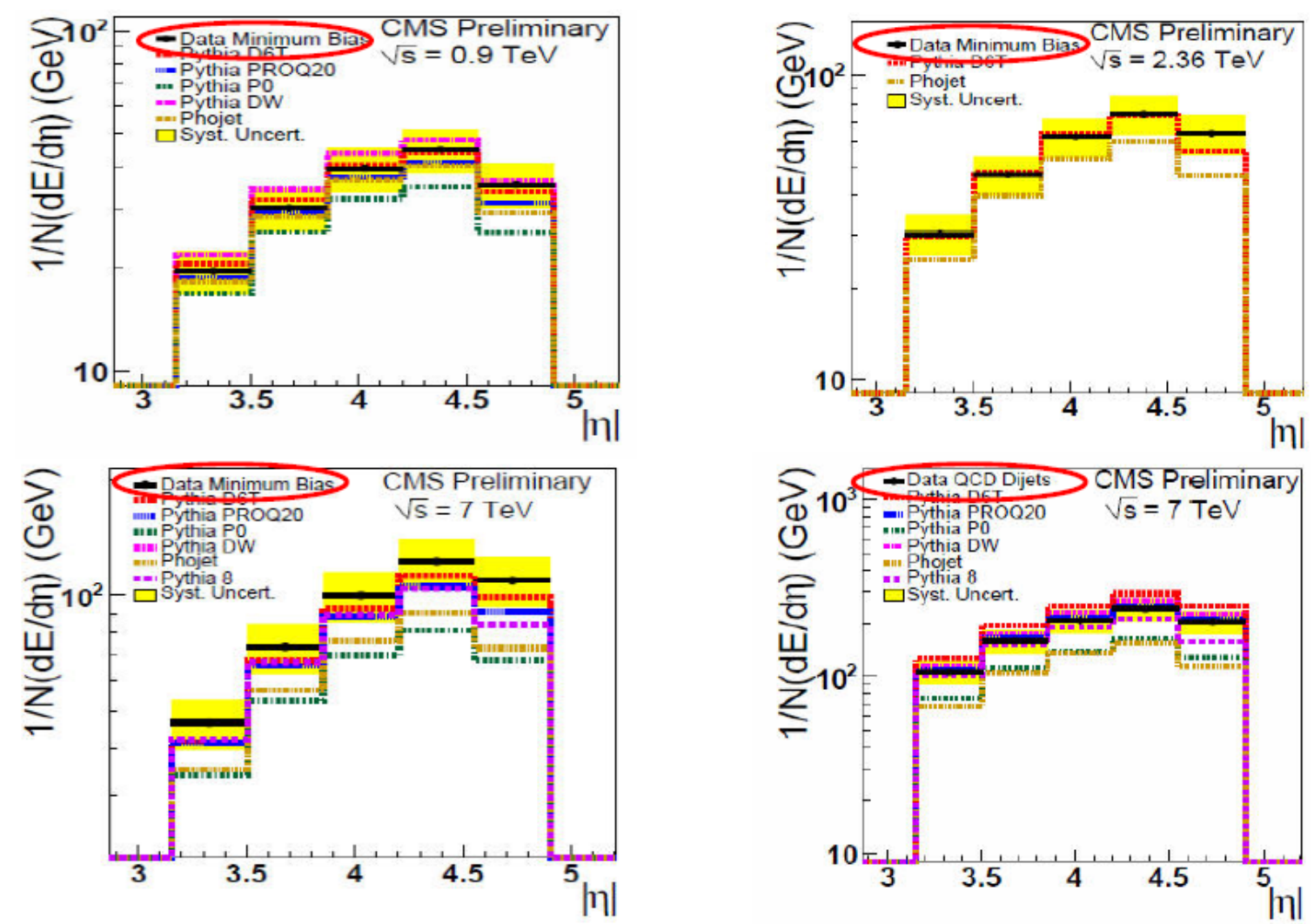

Figure 10: Energy flow in the minimum bias events as a function of pseudorapidity at $\sqrt{s}=900 \mathrm{GeV}$ (top left), $\sqrt{s}=2360 \mathrm{GeV}$ (top right) and at $\sqrt{s}=7000 \mathrm{GeV}$ (bottom left). Energy flow in the di-jet sample at $\sqrt{s}=7000 \mathrm{GeV}$ (bottom right). Uncorrected data are shown as points, the histogram are the predictions from PYTHIA and PHOJET.

A lot has been learnt about small- $x$ physics from measurements at HERA collider. CASTOR along with other CMS forward detectors provides pseudorapidity coverage which was never available before in a collider experiment [6]. Values of Bjorken- $x$ as low as $10^{-5}$ will be reachable. New insights into gluon PDFs are possible in particular looking at forward-central correlations. In a measurement of central jets and a forward jet phase space for additional emissions opens up. This is a way to distinguish between different approaches to parton dynamics. A rough estimate shows that the statistics needed to make the measurement feasible is quite moderate $\left(\sim 1 \mathrm{pb}^{-1}\right)$. Even the worst scenario with CASTOR resolution does not change the picture.

The notion of multi-parton interactions plays an important role in the context of the small- $x$ physics. Forward energy flow measurements are directly sensitive to the amount of parton radiation. They provide important input to determination of parameters for multi-parton interaction models.

CMS performed forward energy flow measurements in the rapidity range covered by the HF calorimeter for 3 different centre-of-mass energies for minimum bias and di-jet events [7]. In Fig. 10 the data are compared to a set of muli-parton interaction tunes. The energy flow is growing with the centre-of-mass energy and no tune is perfect describing the data. Inclusion of CASTOR data is an obvious extension of the analysis toward higher rapidities.

First results for the energy flow measurement with CASTOR are presented in Fig. 11. Total 

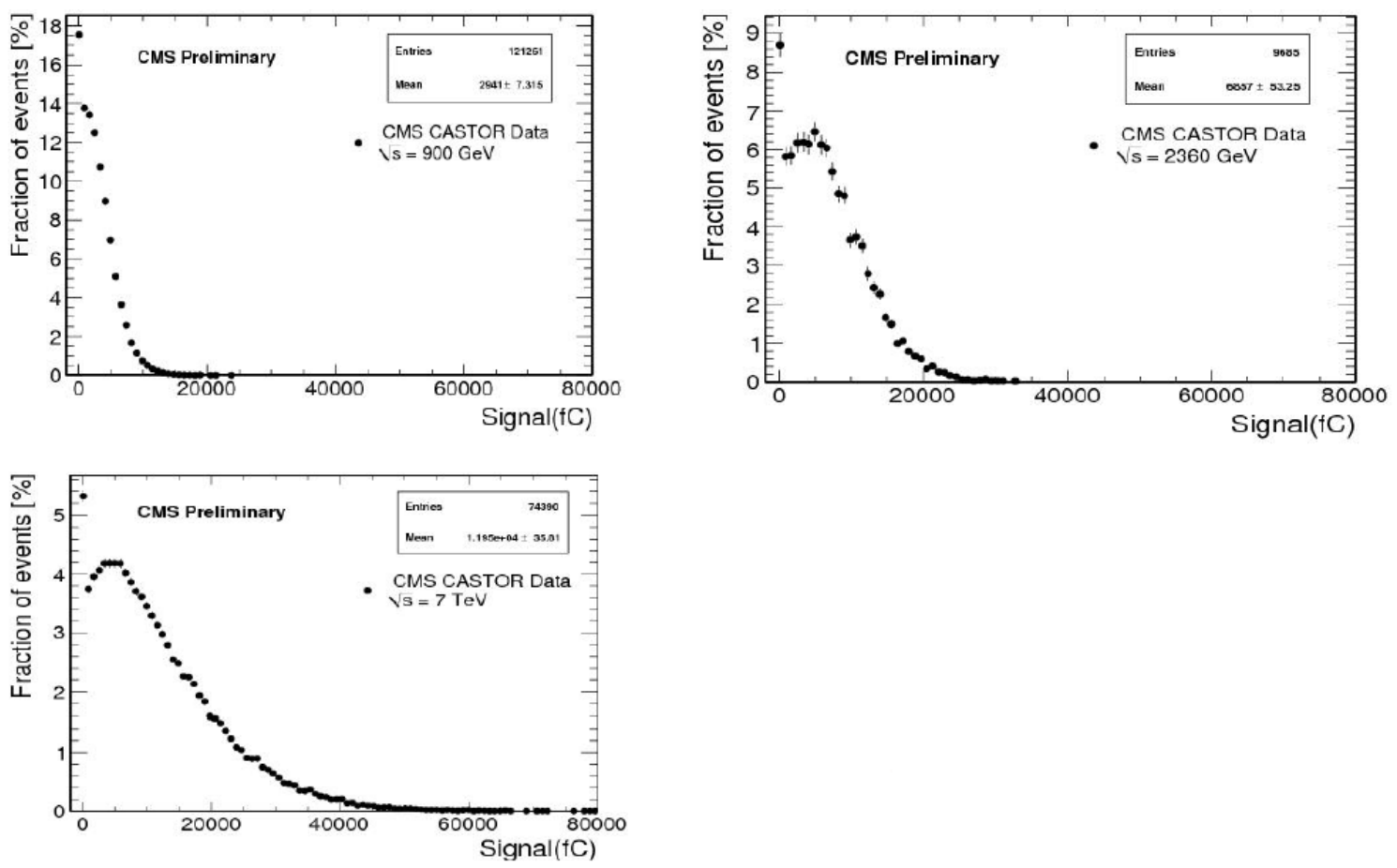

Figure 11: Energy flow in the minimum bias events at $\sqrt{s}=900 \mathrm{GeV}$ (top left), $\sqrt{s}=2360 \mathrm{GeV}$ (top right) and at $\sqrt{s}=7000 \mathrm{GeV}$ (bottom left).

charge collected in the first 5 modules, corresponding to 2.8 interaction lengths, is shown for 3 different centre of mass energies. The energy flow in CASTOR is growing with the centre-of-mass energy. Peaks at zero corresponding to no signal in CASTOR indicate sensitivity to the rapidity gap events.

The CASTOR calorimeter is invaluable for diffractive analyses as a veto detector for event selections based on large rapidity gap requirement due to the well known fact that wider rapidity coverage suppresses non-diffractive events where the gap is caused by a fluctuation. Multiplicity distributions in the forward calorimeters for a combination of diffractive and non-diffractive Monte Carlo are shown in Fig. 12. A clear signal from hard diffractive events in the zero multiplicity bin can be observed already for a moderate statistics.

The name CASTOR stands for Centauro and Strange Object Research and reflects the initial idea to install CASTOR in a heavy-ion experiment to search for exotic objects with unusual longitudinal shower profile properties initially observed in cosmic ray experiments and attracted a lot of interest [9]. CASTOR with its fine longitudinal segmentation and rapidity coverage corresponding to a baryon-rich region is a perfect detector to search for such objects in ultra-relativistic ion-ion collisions at LHC. CASTOR placement is such that along with the CMS zero degree calorimeter it provides additional information for the event centrality determination. Hence a wide range of hot topics of heavy ion physics can be explored.

The LHC offers a unique opportunity to explore the interplay between physics of high energy cosmic rays and accelerator physics in the new energy domain. So far only indirect measurements performed in extensive air showers at $E_{\text {lab }}>10^{15} \mathrm{eV}$ are available as shown in Fig. 13 [10]. The 


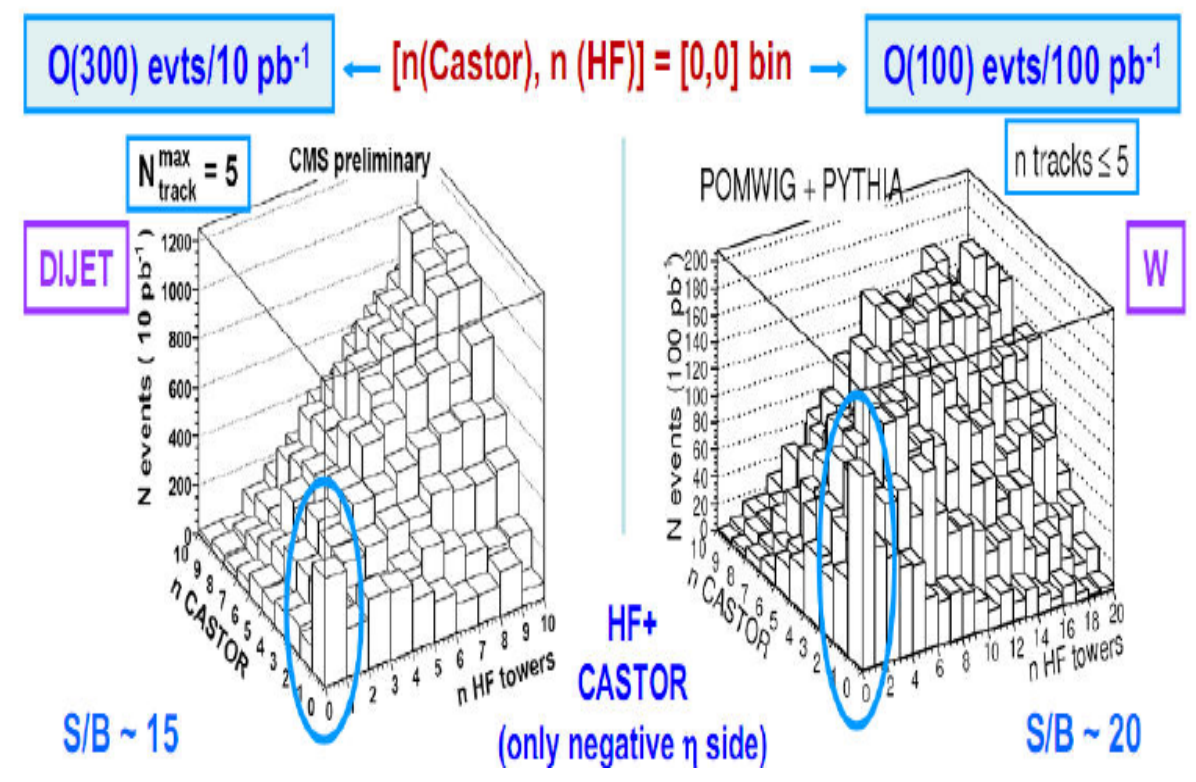

Figure 12: The HF tower multiplicity vs CASTOR sector multiplicity for di-jet (left) and W-production (right) events [8].

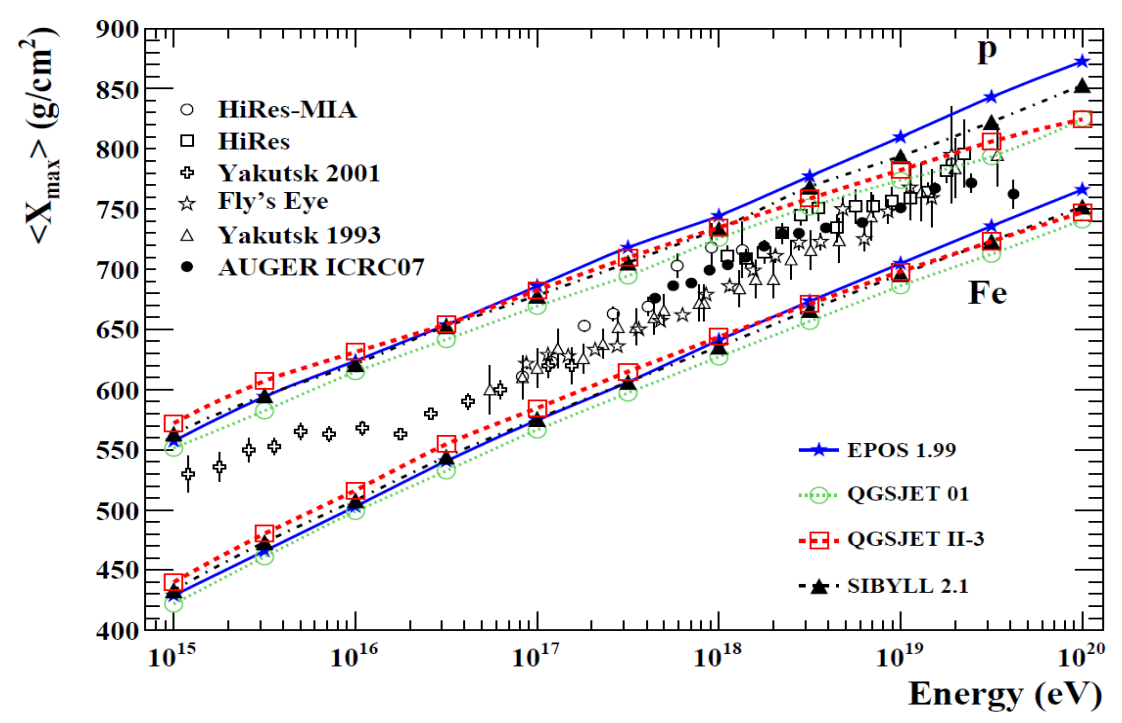

Figure 13: Shower maximum of extensive air showers as a function of primary energy. Predictions of different high-energy hadronic interaction models are compared to data. 
treatment of results in this energy range is strongly dependent on simulations. In particular, conclusions on chemical composition of most interesting ultra high energy cosmic rays suffer from large uncertainty of hadronic interactions modeling. Projectile (pion, proton, nuclei) fragmentation region is of paramount importance for shower development simulations. Accelerator data in this region are scarce. CASTOR calorimeter is placed in the projectile region and hence its measurements are highly valuable for validation of shower simulation codes.

To summarize, despite some obstacles CASTOR, is fully functional and taking high quality data. CASTOR along with other forward detectors is providing the largest rapidity coverage ever achieved in a collider experiment. It substantially enhances CMS physics potential in the wide range of topics from tuning of Monte Carlo generators to potential discovery of exotica. The analysis of data taken with CASTOR is well under way.

\section{Acknowledgments}

We thank the CMS management, engineers and technicians for the support of CASTOR calorimeter project, in particular during the installation and integration phase. The author's work was partly supported by the Helmholtz Association (Germany) and Russian fund for fundamental research (RFFI) under Helmholtz-Russia-Joint-Research Group HRJRG-02.

\section{References}

[1] G. L. Bayatian et al. [CMS Collaboration], CMS technical design report, volume II: Physics performance, J. Phys. G 34 (2007) 995.

[2] P. Goettlicher for the CMS-CASTOR collaboration, Design and test beam studies for the CASTOR calorimeter of the CMS experiment, Nucl. Instrum. Meth. A 623 (2010) 225.

[3] W. Beaumont and G. Antchev for the CMS-CASTOR collaboration, Design of the CMS-CASTOR subdetector readout system by reusing existing designs, Topical Workshop on Electronics for Particle Physics (TWEPP09), Paris, France, (21-25 September 2009).

[4] V. Andreev et al., Performance Studies Of A Full-Length Prototype For The Castor Forward Calorimeter At The Cms Experiment, Eur. Phys. J. C 67 (2010) 601.

[5] O. Ganel and R. Wigmans, On the calibration of longitudinally segmented calorimeter systems, Nucl. Instrum. Meth. A 409 (1998) 621.

[6] M. Albrow et al., Prospects for diffractive and forward physics at the LHC, CERN-LHCC-2006-039.

[7] CMS Collaboration, Measurement of the energy flow at large pseudorapidity at the LHC at $\sqrt{s}=900$, 2360 and $7000 \mathrm{GeV}$, PAS-FWD-10-002 (2010).

[8] M. M. Obertino, Observation of hard diffraction with early CMS data, presented at 17th International Workshop on Deep-Inelastic Scattering (DIS 2009).

[9] E. Norbeck et al. [CMS Collaboration], Exotic physics at the LHC with CASTOR in CMS, CERN-CMS-CR-2007-013.

[10] T. Pierog, R. Engel, and D. Heck, 3D Air Shower Simulations Using CONEX in CORSIKA, to appear in the proceedings of 31st International Cosmic Ray Conference (ICRC 2009). 